\title{
The Angular Momentum of Gas in Protogalaxies. I. Implications for the Formation of Disk Galaxies
}

\section{Citation}

Bosch, Frank C. van den, Tom Abel, Rupert A. C. Croft, Lars Hernquist, and Simon D. M. White. 2002. "The Angular Momentum of Gas in Protogalaxies. I. Implications for the Formation of Disk Galaxies." The Astrophysical Journal 576 (1): 21-35. https://doi.org/10.1086/341619.

\section{Permanent link}

http://nrs.harvard.edu/urn-3:HUL.InstRepos:41381707

\section{Terms of Use}

This article was downloaded from Harvard University's DASH repository, and is made available under the terms and conditions applicable to Other Posted Material, as set forth at http:// nrs.harvard.edu/urn-3:HUL.InstRepos:dash.current.terms-of-use\#LAA

\section{Share Your Story}

The Harvard community has made this article openly available.

Please share how this access benefits you. Submit a story. 


\title{
The Angular Momentum of Gas in Proto-Galaxies: I - Implications for the Formation of Disk Galaxies
}

\author{
Frank C. van den Bosch \\ Max-Planck Institut für Astrophysik, Karl Schwarzschild Str. 1, Postfach 1317, 85741 \\ Garching, Germany \\ vdbosch@mpa-garching.mpg.de \\ Tom Abel ${ }^{1}$, Rupert A. C. Croft ${ }^{2}$, Lars Hernquist \\ Harvard Smithsonian Center for Astrophysics, MA-02138, Cambridge, U.S.A. \\ and \\ Simon D.M. White \\ Max-Planck Institut für Astrophysik, Karl Schwarzschild Str. 1, Postfach 1317, 85741 \\ Garching, Germany
}

\begin{abstract}
We use numerical simulations of structure formation in a Cold Dark Matter (CDM) cosmology to compare the angular momentum distributions of dark matter and non-radiative gas in a large sample of halos. We show that the two components have identical spin parameter distributions and that their angular momentum distributions within individual halos are very similar, all in excellent agreement with standard assumptions. Despite these similarities, however, we find that the angular momentum vectors of the gas and dark matter are poorly aligned, with a median misalignment angle of $\sim 30$ degrees, which might have important implications for spin correlation statistics used in weak lensing studies. We present distributions for the component of the angular momentum that is aligned with the total angular momentum of each halo, and find that for between 5 and 50 percent of the mass this component is negative. This disagrees with the generally adopted 'Universal' angular momentum distribution, for which the
\end{abstract}

\footnotetext{
${ }^{1}$ Present address: Institute of Astronomy, Madingley Road, Cambridge, CB03 0HA, UK

${ }^{2}$ Present address: Physics Department, Carnegie Mellon University, Pittsburgh, PA 15213, U.S.A.
} 
mass fraction with negative specific angular momentum is zero. We discuss the implications of our results for the formation of disk galaxies. Since galactic disks generally do not contain counter-rotating stars or gas, disk formation cannot occur under detailed conservation of specific angular momentum. We suggest that the material with negative specific angular momentum combines with positive angular momentum material to build a bulge component, and show that in such a scenario the remaining material can form a disk with a density distribution that is very close to exponential.

Subject headings: cosmology: dark matter — galaxies: formation - galaxies: structure - galaxies: halos.

\section{Introduction}

Understanding the structure and formation of disk galaxies is intimately linked to understanding the origin of its angular momentum. In hierarchical structure formation scenarios the luminous parts of galaxies form from gas that is cooling and condensing within dark matter (DM) halos and these merge to build larger and larger objects (White \& Rees 1978). Within this picture, the current paradigm for disk formation contains three important ingredients: (i) the angular momentum originates from cosmological torques (Hoyle 1953), (ii) the gas and dark matter within virialized systems have initial angular momentum distributions that are identical (Fall \& Efstathiou 1980), and (iii) the gas conserves its specific angular momentum when cooling (Mestel 1963).

It is well established that cosmological torques impart angular momentum to dark matter halos. Many studies have investigated the resulting angular momenta of dark matter halos using either tidal torque theory (e.g., Peebles 1969; Doroshkevich 1970; White 1984; Catelan \& Theuns 1996) or N-body simulations (e.g., Barnes \& Efstathiou 1987; Warren et al. 1992; Bullock et al. 2001). The specific angular momentum of halos is typically parameterized by the dimensionless spin parameter

$$
\lambda \equiv \frac{|\mathbf{J}||E|^{1 / 2}}{G M^{5 / 2}},
$$

with $G$ the gravitational constant and $\mathbf{J}, E$, and $M$ the total angular momentum, energy and mass of the object, respectively (Peebles 1969). Under the assumptions (ii) and (iii) disks have scale lengths $R_{d} \propto \lambda R_{\text {vir }}$. Given the distributions of halo spin parameters and of halo virial radii $R_{\text {vir }}$, the implied distribution of disk scale lengths is in excellent agreement with

observations (e.g., Dalcanton, Spergel \& Summers 1997; Mo, Mao \& White 1998; de Jong 
\& Lacey 2000). This success has prompted many detailed studies of disk galaxy formation, always under the three assumptions listed above (van den Bosch 1998, 2000, 2001, 2002; Jimenez et al. 1998; Natarajan 1999; Heavens \& Jimenez 1999; Firmani \& Avila-Reese 2000; Avila-Reese \& Firmani 2000; Buchalter, Jimenez \& Kamionkowski 2001).

The standard picture of disk formation that has emerged from these studies has been remarkably successful in explaining a wide variety of observational properties of disk galaxies. However, two significant problems, both related to the angular momentum, have come to light. First of all, detailed hydro-dynamical simulations of disk formation in a cold dark matter (CDM) Universe yield disks that are an order of magnitude too small (Navarro \& Benz 1991; Navarro \& White 1994; Steinmetz \& Navarro 1999; Navarro \& Steinmetz 2000). This problem, known as the angular momentum catastrophe, is a consequence of the hierarchical formation of galaxies which causes the baryons to lose a large fraction of their angular momentum to the dark matter. It is unclear whether this is a problem for the theory or merely for the particular way in which feedback processes are implemented (or ignored) in the simulations (e.g., Navarro \& White 1994; Weil, Eke \& Efstathiou 1998; Sommer-Larsen, Gelato \& Vedel 1999; Thacker \& Couchman 2001).

The second problem concerns the actual density structure of galaxy disks. Assuming detailed angular momentum conservation, this structure is a direct reflection of the distribution of specific angular momentum in the proto-galaxy. Bullock et al. (2001) determined the angular momentum distributions of individual dark matter halos, which according to assumption (ii) should also reflect the angular momentum distribution of the gas that forms the disk. However, these distributions seem to have far too much low angular momentum material to be consistent with the typical exponential density distributions of disk galaxies (Bullock et al. 2001; van den Bosch 2001; van den Bosch, Burkert \& Swaters 2001). Knebe et al. (2002) and Bullock, Kravtsov \& Colín (2002) investigated the angular momentum profiles of warm dark matter (WDM) halos. Except for a slight difference in the average spin parameter, CDM and WDM halos have very similar angular momentum distributions, implying that this particular problem cannot be solved by invoking WDM instead of CDM. A more promising solution has recently been suggested by Maller \& Dekel (2002). In their picture halo angular momentum originates from the orbital angular momentum of merging satellites, rather than from pure tidal torques (see also Maller, Dekel \& Somerville 2001). Combining this picture with a simple model for feedback and tidal stripping Maller \& Dekel are able to explain the angular momentum profiles of dwarf galaxies measured by van den Bosch et al. (2001).

These angular momentum problems seem to imply that assumptions (ii) and (iii) listed above, and which have been essential ingredients of our standard paradigm for the formation 
of disk galaxies, may be incorrect. Remarkably, despite many papers that have investigated the angular momentum distributions within dark matter halos, to the best of our knowledge, no study so far has addressed the spin distribution of the gas in these halos in order to test whether the ansatz that the gas and dark matter have similar initial distributions of specific angular momentum is correct. In fact, it seems plausible that they may not. Gas and dark matter suffer different relaxation mechanisms during halo collapse: Whereas the dark matter undergoes collisionless virialization through violent relaxation, the gas attempts to achieve a hydrostatic equilibrium through shocks. In order to test whether the initial angular momentum distributions of gas and dark matter are indeed similar, we perform numerical simulations of structure formation in a $\Lambda$ CDM cosmology including both dark matter and a non-radiative gas. After presenting our simulations and outlining our analysis methods (Section 2), we present various statistics of the angular momentum distributions (Section 3). In Section 4 we discuss the implications of our results for the theory of disk galaxy formation, and we summarize our results in Section 5.

\section{Method}

\subsection{Numerical Simulations}

We use the public version of the smoothed particle hydrodynamics (SPH) code GADGET developed by V. Springel (1999) and described in Springel, Yoshida \& White (2000). The chosen flat cosmology is vacuum energy dominated with $\Omega_{\Lambda}=0.7$ and matter content $\Omega_{m}=0.3$ with a baryon density $\Omega_{b}=0.021 h^{-2}$ and a Hubble constant of $67 \mathrm{~km} \mathrm{~s}^{-1} \mathrm{Mpc}^{-1}$ (i.e., $h=0.67$ ). We choose a relatively small box of $10 h^{-1}$ comoving Mpc and evolve it from redshift 59 down to redshift three. We use identical power-spectra for baryons and dark matter given by the fitting formula of Efstathiou, Bond \& White (1992) and use $128^{3}$ particles each for the gas and the dark matter component. The power spectrum is normalized to have a typical mean overdensity of $\sigma_{8}=0.9$ at redshift zero in a top hat sphere of $8 h^{-1} \mathrm{Mpc}$. Gas and dark matter particles have masses of $m_{\text {gas }}=6.26 \times 10^{6} h^{-1} \mathrm{M}_{\odot}$ and $m_{\mathrm{DM}}=3.34 \times 10^{7} h^{-1} \mathrm{M}_{\odot}$, respectively. The gravitational softening lengths for both the gas and the dark matter were chosen to be $4 h^{-1} \mathrm{kpc}$ (comoving). The gas has an initial entropy corresponding to a temperature of $2 \times 10^{4} \mathrm{~K}$ for a mean molecular weight of one proton mass. An artificial temperature floor at $10^{4} \mathrm{~K}$ (for the same molecular weight) is introduced to mimic the heating effects from re-ionization. Both this temperature floor and the initial temperature are too low to play a significant role in the simulated dynamics because they are significantly lower than the virial temperatures of the halos we analyze. This is evident in our analysis as the reported results do not depend on the mass of the halos studied. All 
results presented below correspond to the final output at $z=3$.

The robustness of our results with respect to resolution, simulation box size, and the redshift of analysis is addressed in Section 3.3.

\subsection{The Halo Identification Algorithm}

We start by using the publicly available group finder HOP, written by Eisenstein \& Hut (1998), to identify overdensity peaks in the dark matter distribution using a threshold density of 20 times the mean dark matter density. In each group we identify the densest particle, and we determine the virial radius $R_{\text {vir }}$ of the spherical volume, centered on this densest particle, inside of which the average dark matter density is $\Delta_{\text {vir }}(z)$ times the critical density $\rho_{\text {crit }}(z)$. For our adopted cosmology and at the redshift of the output analyzed $\Delta_{\text {vir }}=174.7$ (Bryan \& Norman 1998). Next we compute the center-of-mass of all dark matter particles inside this spherical volume which we associate with the new center of the halo. We compute the new $R_{\text {vir }}$ around this new halo center, as well as the associated new center-of-mass. This procedure is iterated until the distance between the center-of-mass and the adopted halo center is less than one percent of the virial radius $R_{\text {vir }}$. Typically this requires of the order of 2 to 5 iteration steps.

All dark matter and gas particles inside the resulting spherical volume with radius $R_{\text {vir }}$ are considered halo members. Although our iterative method assures that the center-ofmass of the dark matter component is similar to the adopted halo center, the same is not necessarily true for the gas component. We therefore remove all halos from our sample for which the distance between the halo center and the center-of-mass of the gas particles is larger than 10 percent of $R_{\text {vir }}$. We checked that none of our results correlate with the offset between the centers of mass of the gas and dark matter. In addition, we repeated the entire analysis adopting the position of the most bound particle as halo center and found very simular results.

In order to allow sufficiently accurate measurements of the angular momentum vectors of the gas and dark matter we only accept halos that have more than 100 gas particles and more than 100 dark matter particles. In addition, if any two halos overlap, i.e., the distance between the halo centers is less than the sum of their virial radii, we remove the least massive halo from our sample. This leaves a total of 378 halos of which 259 are isolated in that they have no subgroups detected, and they do not overlap with any other halo. Of these 378 halos

23 have more than 5000 particles (in total), and are used for a more detailed analysis of the distribution of specific angular momentum (see Section 3.2). 


\subsection{Theoretical Background}

Our main goal is to compare the angular momentum distributions of the gas and dark matter components. However, one cannot do so by simply computing angular momentum distributions from the velocities of the gas and dark matter particles. Both the dark matter and the gas are fluids, for which the velocity of each microscopic particle can be written as $\mathbf{v}=\mathbf{u}+\mathbf{w}$. Here $\mathbf{u}$ is the mean streaming motion at the location $\mathbf{x}$ of the microscopic particle, and $\mathbf{w}$ is the particle's random motion. The macroscopic, collisionless dark matter particles in $N$-body simulations can be considered a Monte Carlo realization of the phasespace distribution function of all microscopic particles. The velocities of these particles in the simulation correspond to $\mathbf{v}$ and are computed by solving Newton's equations of motion. In the case of the gas, however, the Smoothed Particle Hydrodynamics (SPH) approach is used, which yields the streaming motions $\mathbf{u}$ of the gas particles by solving the Euler equations (with some artificial viscosity to take account of shocks). Information about the random motion of the gas particles is provided by the internal energy of each particle.

Thus the velocities of the dark matter particles and gas particles in the simulation correspond to different motions. This must be taken into account before comparing their angular momentum distributions. This is most easily achieved by computing the velocities $\mathbf{v}$ of the gas particles from their streaming motions and their (isotropic) velocity dispersion tensor. For each gas particle we randomly draw 100 random velocities w which we add to the particle's streaming motion u. For each of the three Cartesian components of w we draw a velocity from a Gaussian with a standard deviation given by

$$
\sigma=\sqrt{\frac{k T}{\mu}}=\sqrt{\frac{2 U}{3}}
$$

Here $U$ and $T$ are the internal energy per unit mass and temperature of the gas particle, respectively, $k$ is Boltzmann's constant, and $\mu$ is the mean molecular weight of the gas. After this 'thermal broadening' we find that the rms velocities $\left\langle|\mathbf{v}|^{2}\right\rangle$ of the gas and dark matter particles are, for the vast majority of our halos, very similar, indicating that the halos are, to a good approximation, in hydrostatic equilibrium.

We thus use the velocities $\mathbf{v}$ to compare the angular momentum distributions of the gas and dark matter. However, the distribution that is of interest for galaxy formation is the angular momentum distribution based on the streaming motions $\mathbf{u}(\mathbf{x})$. After all, when the gas cools $\mathbf{v} \rightarrow \mathbf{u}$, i.e., the typical random motions become negligible, at least in sufficiently massive halos. Under the assumption that the gas conserves its specific angular momentum, the resulting disk will thus have an angular momentum distribution related to its streaming

motions $\mathbf{u}$. Ideally one would therefore like to compare the angular momentum distributions 
computed from the streaming motions $\mathbf{u}(\mathbf{x})$. However, this requires estimating the streaming motions of the dark matter. In principle this could be achieved by smoothing the dark matter velocities $\mathbf{v}$, but it is unclear what smoothing scale to use and resolution issues are likely to play an important role. We therefore do not attempt to compute the dark matter streaming motions, but instead use the velocities $\mathbf{v}$ of the dark matter and gas particles when comparing their angular momentum distributions. However, the streaming motions of the gas are available, and we present a detailed comparison of the angular momentum distributions of the gas based on $\mathbf{v}$ and $\mathbf{u}$ in Section 3.2.

\subsection{Analysis}

For each halo we compute the total angular momenta of the gas and the dark matter particles, using,

$$
\mathbf{J}_{\text {gas }, \mathrm{DM}}=m_{\text {gas }, \mathrm{DM}} \sum_{i=1}^{N_{\mathrm{gas}, \mathrm{DM}}} \mathbf{r}_{i} \times\left(\mathbf{v}_{i}-\overline{\mathbf{v}}_{\mathrm{com}}\right) .
$$

Here $\overline{\mathbf{v}}_{\text {com }}$ is the mean (bulk) velocity of the entire halo (dark matter plus gas) and $\mathbf{r}_{i}$ is the radial vector with respect to the halo center. We follow Bullock et al. (2001) and define the following modified spin parameters

$$
\lambda_{\text {gas }, \mathrm{DM}}^{\prime}=\frac{\left|\mathbf{j}_{\text {gas }, \mathrm{DM}}\right|}{\sqrt{2} R_{\mathrm{vir}} V_{\mathrm{vir}}} .
$$

Here $\mathbf{j}_{\text {gas }}$ and $\mathbf{j}_{\mathrm{DM}}$ are the specific angular momenta of the gas and dark matter, respectively, and $V_{\text {vir }}=\sqrt{G\left(M_{\text {gas }}+M_{\mathrm{DM}}\right) / R_{\mathrm{vir}}}$ is the circular velocity at the virial radius $R_{\mathrm{vir}}$. This definition of the spin parameter has the advantage that it does not depend on the energy content of the halo which can be difficult to estimate reliably. In what follows we always adopt the spin parameter definition of equation (4), and we drop the prime.

In addition to the spin parameters of the dark matter and the gas we also compute the angle

$$
\theta=\cos ^{-1}\left[\frac{\mathbf{J}_{\text {gas }} \cdot \mathbf{J}_{\mathrm{DM}}}{\left|\mathbf{J}_{\text {gas }}\right|\left|\mathbf{J}_{\mathrm{DM}}\right|}\right]
$$

between the angular momentum vectors of the two components. After rotating the coordinate frame such that the $z$-axis is aligned with the angular momentum vector of either the dark matter or the gas, we also compute the fractions of dark matter and gas particles with negative angular momentum about their respective $z$-directions. These fractions are denoted by $f_{\mathrm{DM}}$ and $f_{\text {gas }}$, respectively, and give an indication of the amount of disordered motion relative to the ordered motion. 
Note that since at each position the expectation value of $\mathbf{w}$ is, by definition, the null vector, the values of $\lambda_{\text {gas }}, \lambda_{\mathrm{DM}}$ and $\theta$ are independent of whether one considers the streaming motions $\mathbf{u}$ or the microscopic velocities $\mathbf{v}$. However, the angular momentum distributions may be quite different. In order to distinguish these two cases we label the appropriate quantities with the superscript $v$ whenever the microscopic velocities $\mathbf{v}$ are used. Thus a particle's specific angular momentum is given by either $\mathbf{j}=\mathbf{r} \times \mathbf{u}$ or $\mathbf{j}^{v}=\mathbf{r} \times \mathbf{v}$.

\section{Results}

Figure 1 plots the histograms of $\lambda_{\mathrm{DM}}$ and $\lambda_{\text {gas }}$ for all 378 halos in our sample. Both distributions are well fit by a log-normal distribution

$$
p(\lambda) \mathrm{d} \lambda=\frac{1}{\sqrt{2 \pi} \sigma_{\lambda}} \exp \left(-\frac{\ln ^{2}(\lambda / \bar{\lambda})}{2 \sigma_{\lambda}^{2}}\right) \frac{\mathrm{d} \lambda}{\lambda} .
$$

For the dark matter we find $\bar{\lambda}_{\mathrm{DM}}=0.040$ and $\sigma_{\lambda_{\mathrm{DM}}}=0.56$, while for the gas $\bar{\lambda}_{\text {gas }}=0.039$ and $\sigma_{\lambda_{\text {gas }}}=0.57$. Clearly, the distributions of $\lambda_{\text {gas }}$ and $\lambda_{\mathrm{DM}}$ are indistinguishable. If we only consider the 259 isolated halos we obtain very similar distributions both for the dark matter and for the gas.

In the upper left panel of Figure 2 we plot $\lambda_{\text {gas }}$ versus $\lambda_{\mathrm{DM}}$. Although $\lambda_{\text {gas }}$ and $\lambda_{\mathrm{DM}}$ are well correlated, with a Spearman rank coefficient $r_{s}=0.67$, which has a probability of virtually zero to occur under the null hypothesis of no correlation, the scatter is relatively large. The average of $\lambda_{\text {gas }}-\lambda_{\mathrm{DM}}$ is -0.001 with a standard deviation of 0.020 , which confirms once again that on average the spin parameters of the gas and dark matter are very similar. However, on a one-to-one basis the two components can have quite different specific angular momenta; i.e., the average (median) of $\left|\lambda_{\text {gas }}-\lambda_{\mathrm{DM}}\right|$ is 0.015 (0.011). We find no significant trend between $\lambda_{\text {gas }}-\lambda_{\mathrm{DM}}$ and $M_{\mathrm{vir}}$ (see upper left panel of Figure 4) indicating that the scatter is real, and not an artifact of discreteness noise.

The upper right panel of Figure 2 plots $f_{\text {gas }}^{v}$ versus $f_{\mathrm{DM}}^{v}$. With $\left\langle f_{\mathrm{DM}}^{v} / f_{\text {gas }}^{v}\right\rangle=0.97 \pm 0.10$ the two mass components have very similar mass fractions with negative specific angular momentum. The middle panels of Figure 2 show that the values of $f_{\text {gas }}^{v}$ and $f_{\mathrm{DM}}^{v}$ are strongly anti-correlated with their respective spin parameters. A similar anti-correlation is also present for the gas when $f_{\text {gas }}$, is used instead of $f_{\text {gas }}^{v}$ (lower left panel). Again, none of these results change if we only focus on the 259 isolated halos. These anti-correlations indicate that it is the amount of ordered motion that is directly responsible for the amplitude of the total specific angular momentum.

Finally, the lower right panel of Figure 2 plots $f_{\text {gas }}$ versus $f_{\text {gas }}^{v}$. For all halos $f_{\text {gas }}<f_{\text {gas }}^{v}$, 
illustrating the trivial result that the streaming motions of the gas are more ordered than their microscopic motions. Note, however, that there is not simply a constant offset between $f_{\text {gas }}$ and $f_{\text {gas }}^{v}$. Rather, the difference between $f_{\text {gas }}$ and $f_{\text {gas }}^{v}$ is larger for halos with larger $\lambda_{\text {gas }}$, and the distribution of $f_{\text {gas }}$ is much broader than that of $f_{\text {gas }}^{v}$. All these results have implications for the formation of disk galaxies, as we discuss in more detail in Section 4 .

\subsection{Misalignment of Angular Momentum Vectors}

Figure 3 plots the distribution of the misalignment angle $\theta$ (in degrees) between the total angular momentum vectors of the dark matter and the gas. The mean $\left(\sim 36^{\circ}\right)$ and median $\left(\sim 27^{\circ}\right)$ of the distribution are similar for the entire sample and for the subsample of isolated halos.

Contrary to what one might expect, there is no correlation between the misalignment angle $\theta$ and the absolute difference between $\lambda_{\text {gas }}$ and $\lambda_{\mathrm{DM}}$. This is evident from the upper right panel of Figure 4. The solid lines indicate the mean and the 68 percent interval of $\theta$. We do, however, find a small trend that the average misalignment angle increases with decreasing halo mass and spin parameter (see lower panels of Figure 4). We stress that there is no correlation between $M_{\text {vir }}$ and $\lambda_{\text {gas }}$ so that these are two independent trends. Both are likely due to discreteness effects, in that the direction of the angular momentum vectors can be more accurately determined if either the total angular momentum or the number of halo particles is larger. However, since both trends are weak and the average misalignment angle is still significantly different from zero even for the most massive halos and for the ones with the largest spin parameters, we conclude that there is a true misalignment between the angular momenta of the gas and the dark matter (e.g., see column (9) of Table 1).

This misalignment may have important implications for galaxy spin correlation statistics. In the past, both tidal torque theory (Crittenden et al. 2001; Heavens, Refegier \& Heymans 2000; Natarajan et al. 2001) and pure N-body simulations (Croft \& Metzler 2000) have been used to calculate the possibility of intrinsic alignments of galaxy shapes, which may be responsible for part of the measured weak lensing signal (e.g. van Waerbecke et al. 2000; McKay et al. 2001), and may be used to reconstruct the shear field (Lee \& Pen 2000, 2001). In these studies the assumption is made that the angular momentum vectors of the galaxies are aligned with those of the dark matter halos. The relatively large misalignment angles found here, however, may diminish any alignment of galaxy shapes that one might expect based on tidal torque theory. We emphasize, however, that simulations of higher resolution are required to reduce the possible impact of discreteness effects on the distribution of misalignment angles. 


\subsection{The Distribution of Angular Momentum}

Although $\sim 100$ particles is enough to obtain a reliable estimate of the spin parameter, many more particles are required to determine angular momentum distributions (hereafter AMDs) within individual haloes. We therefore limit ourselves here to halos with more than 5000 particles in total, of which there are 23 in our sample.

We define the normalized specific angular momentum $l \equiv j /\left(R_{\text {vir }} V_{\text {vir }}\right)$ and compute separate distributions $p(l)$ for dark matter particles and for gas particles. Here and in what follows $j$ refers to the specific angular momentum along the $z$-axis. Note that $R_{\text {vir }} V_{\text {vir }}$ is the maximum specific angular momentum that a halo particle can have (i.e., $l \in[-1,1])$. We normalize $p(l)$ to unity so that

$$
\lambda=\frac{1}{\sqrt{2}} \int_{-1}^{1} p(l) l \mathrm{~d} l
$$

In Figure 5 we plot $p\left(l^{v}\right)$ both for the gas (dashed lines) and for the dark matter (solid lines) of 20 halos that are selected randomly from the sample of 23. Some global parameters of these 20 halos are listed in Table 1. As can be seen, the AMDs of the two mass components are remarkably similar. The fact that the $p\left(l^{v}\right)$ of the gas appear less noisy than for the dark matter is a consequence of the fact that because of the 'thermal broadening' of the gas velocity field we effectively have on average 100 times more gas particles per halo than dark matter particles (see Section 2.3). In a few cases small differences can be discerned, but there is no indication for any systematic difference between the AMDs of the two components. Therefore, the standard assumption that the gas and dark matter have the same distributions of specific angular momentum is to first order correct, and the shocks that occur during the virialization process do not significantly modify the angular momentum distribution of the gas.

In Figure 6 we compare the gas AMD $p\left(l^{v}\right)$ (dashed lines) to the distribution $p(l)$ as computed from the streaming motions of the gas (solid lines). Here a clear systematic difference is apparent; typically $p(l)$ has a less extended wing to negative specific angular momentum than $p\left(l^{v}\right)$ (cf. lower right panel of Figure 2), and a more pronounced peak at low angular momentum. Note that with equation (7) both distributions integrate to the same value of $\lambda_{\text {gas }}$.

In Figures 7 and 8 we plot $\lambda(R)$ and $\Delta \theta(R)$ as functions of $R / R_{\text {vir }}$, respectively. Results for both the dark matter (solid lines) and the gas (dashed lines) are shown. Here $\lambda(R)$ is the spin parameter inside a sphere of radius $R$, i.e.,

$$
\lambda(R)=\frac{j^{v}(R)}{\sqrt{2 G R M(R)}}
$$


with $j^{v}(R)$ and $M(R)$ the specific angular momentum and mass inside a sphere of radius $R$, respectively. $\Delta \theta(R)$ is defined as the angle between the angular momentum vector inside radius $R$ and that inside $R_{\text {vir }}$ (i.e., by definition $\left.\Delta \theta\left(R_{\text {vir }}\right)=0\right)$. In some cases the gas and dark matter have fairly similar $\lambda(R)$ and $\Delta \theta(R)$ profiles (e.g., halos 14, 19, and 58), while in other cases the gas and dark matter seem to have quite distinct profiles (e.g., halos 36, 156, 582). Remarkably, halos for which the $\lambda(R)$ and/or $\Delta \theta(R)$ profiles are quite different for the gas and dark matter are not necessarily also the ones that have the least similar AMDs, nor do they correspond to halos for which any of the global parameters listed in Table 1 are in any way special. Note that $\lambda$ typically either decreases or stays roughly constant with radius, while the angular momentum directions of both the gas and the dark matter typically change quite dramatically from the center to the virial radius. We discuss the implications of this intrinsic misalignment for the formation of disk galaxies in Section 4.

\subsection{Robustness of results}

The results presented above are based on a numerical simulation with relatively low resolution ( $128^{3}$ particles), with a small box size $\left(L_{\text {box }}=10 h^{-1} \mathrm{Mpc}\right.$ comoving), and which has only been run to redshift $z=3$. Too small resolution causes shocks to be poorly resolved, which could result in an underestimate of the possible angular momentum redistribution of the gas during its relaxation process. A small box size may cause an inaccurate representation of the cosmological tidal field responsible for the angular momentum of halos. Finally, it is not a priori clear that angular momentum distributions at $z=3$ are representative for those at $z=0$.

In order to address the robustness of our results against these effects we analyze a high resolution numerical simulation of a cluster kindly provided by Naoki Yoshida. In this simulation exactly the same cosmological parameters are adopted as in the simulation discussed above (see Section 2.1). It is based on the technique of 'zooming in' on a region of interest (Tormen, Bouchet \& White 1997). The parent simulation from which this region is chosen is the GIF- $\Lambda$ CDM simulation of Kauffmann et al. (1999) which followed $256^{3}$ particles within a comoving box with $L_{\text {box }}=141.3 h^{-1} \mathrm{Mpc}$. The Lagrangian region that collapses to form the second most massive halo in this GIF simulation (with virial mass $\sim 8 \times 10^{14} h^{-1} \mathrm{M}_{\odot}$ ) has been resimulated with greatly increased mass and force resolution and including a nonradiative gas. This high resolution region is represented by $\sim 2.2 \times 10^{5}$ dark matter and gas particles each, with masses of $1.2 \times 10^{10} h^{-1} \mathrm{M}_{\odot}$ and $2.1 \times 10^{9} h^{-1} \mathrm{M}_{\odot}$, respectively. Outside this high-resolution region $\sim 3.1 \times 10^{6}$ particles with larger masses are used to represent the cosmological tidal field. More details regarding this simulation can be found in Springel et 
al. (2001) and Yoshida et al. (2000a,b)

We analyze the angular momentum distributions of the dark matter and gas of this high resolution halo at $z=0$. Using the standard friends-of-friends algorithm (Davis et al. 1985) we identify the most massive group and its most bound particle. Next the virial radius $R_{\text {vir }}$ of the spherical volume, centered on this particle, is determined adopting $\Delta_{\text {vir }}=101$ (appropriate for this cosmology and redshift; Bryan \& Norman 1998). The halo thus defined (hereafter referred to as halo 'S0') contains $\sim 1.2 \times 10^{5}$ particles (more than twice that of the biggest halo analyzed in the previous section), and has a virial mass and radius of $M_{\text {vir }}=8.7 \times 10^{14} h^{-1} \mathrm{M}_{\odot}$ and $1.86 h^{-1} \mathrm{Mpc}$, respectively. Analyzing the angular momentum distributions of the dark matter and gas particles as in Section 2.4 we obtain the spin parameters and mass fractions with negative specific angular momentum listed in Table 1. The resulting angular momentum distributions $p\left(l^{v}\right)$ and $p(l)$, as well as $\lambda(R)$ and $\Delta \theta(R)$, are shown in Figure 9.

Note that the angular momentum distributions of halo 'S0' are in no way systematically different from those of the sample of halos analyzed above. In particular, once again we find that the gas and dark matter have virtually identical angular momentum distributions $p\left(l^{v}\right)$, and that a large mass fraction $(\sim 45$ percent) of the gas has negative specific angular momentum. The $\lambda(R)$ and $\Delta \theta(R)$ profiles are similar to that of halo 11 (cf. Figures 7 and 8). Although one has to be careful to draw conclusions based on a single high resolution halo, these results suggest that the characteristics of the angular momentum distributions presented in Section 3.2 are not influenced by the relatively low resolution, by the small box size, or by the fact that we analyzed the halos at $z=3$.

\subsection{Do Dark Matter Halos follow a Universal Angular Momentum Profile?}

Bullock et al. (2001; hereafter B01) computed the angular momentum distributions of dark matter halos and argued that these follow a universal profile given by:

$$
P(j) \equiv \frac{M(<j)}{M_{\text {vir }}}=\frac{\mu\left(j / j_{\max }\right)}{\left(j / j_{\max }\right)+\mu-1},
$$

Here $M(<j)$ is the halo mass with specific angular momentum less than $j, j_{\max }$ is the maximum specific angular momentum of the entire AMD, and $\mu$ is a free fitting parameter. Note that $P(j)$ corresponds to the cumulative distribution of the specific angular momentum. In order to compute these AMDs, B01 split up their halos in a number of spatial cells. The angular momentum profiles $P(j)$ are then constructed by ranking the cells according to their

total specific angular momentum $j$ from which the best-fitting value of $\mu$ is determined. Note 
that this cell averaging should in principle average out the random motions $\mathbf{w}$ of the dark matter particles, and equation (9) should thus be interpreted as the cumulative distribution of $p(j)$ rather than $p\left(j^{v}\right)$.

However, there are two problems. First of all, it is unlikely that the particular cell geometry adopted by B01 yields the true streaming motions of the dark matter (see the discussion in Section 2.3). This means that, depending on the actual cell geometry adopted, the AMD of equation (9) does not necessarily reflect the true $p(j)$. Secondly, because $j$ corresponds to a projected component the total $j$ of any cell can be negative. B01 ignored this and removed all cells with negative $j$ from their ranked list. We find that the $p(l)$ of our halos are well fit by equation (9) for $l \geq 0$, in agreement with B01. However, whereas B01 have implicitely assumed that there is no mass with negative specific angular momentum, we find large mass fractions with $l<0$, at least for the gas. Since measuring the true streaming motions of the dark matter is non-trivial we have not attempted to obtain the $p(l)$ for the dark matter. However, since the $p\left(l^{v}\right)$ of the gas and dark matter are virtually identical, it seems reasonable to assume that the same applies for the $p(l)$-distributions ${ }^{3}$. If confirmed, between $\sim 5$ and 50 percent of the dark matter mass has negative specific angular momentum, in clear disagreement with the 'Universal' profile of B01. Furthermore, since the mass fraction with negative specific angular momentum is strongly anti-correlated with the total specific angular momentum, the 'Universal' angular momentum profile of equation (9) lacks an essential characteristic of the true AMDs.

\section{Implications of the Formation of Disk Galaxies}

In the standard picture of disk formation, (i) the specific angular momentum of disk galaxies arises from cosmological torques, (ii) is identical to that of their dark matter halos, and (iii) is conserved during the process of disk formation. Recently several authors have pointed out that this picture is inconsistent with observations, as it would result in disks that are too centrally concentrated (B01; van den Bosch 2001; van den Bosch et al. 2001). This is a reflection of the fact that the specific angular momentum distributions of dark matter halos, as described by the 'Universal' profile of B01, contain relatively too much low angular momentum material.

As we argued in Section 1 this seems to suggest that one or more of the standard assumptions is incorrect. Our results clearly show that in the non-radiative case the angular

\footnotetext{
${ }^{3}$ The accuracy of this assumption needs to be checked using simulations of higher resolution than the one analyzed here.
} 
momentum distributions of the gas and dark matter are virtually identical, thereby lending strong support to (ii). However, our results also indicate that assumption (iii) cannot be correct. As shown, the angular momentum distributions $p(l)$ of the gas contain relatively large mass fractions with negative specific angular momentum. Disk galaxies, on the other hand, generally do not contain material with negative specific angular momentum. This suggests that detailed conservation of specific angular momentum has to be violated if one wants to build a disk out of the typical AMDs of the gas in proto-galaxies.

Once the gas starts to cool, its density increases, enhancing the frequency of shocks through which the gas parcels exchange angular momentum. Gas parcels with negative $j_{z}$ can share their angular momentum with parcels with positive $j_{z}$, thus increasing the mass fraction with low angular momentum. This, however, can only aggravate the low angular momentum problem. Apparently real disks do not contain a large fraction of low angular momentum material (van den Bosch et al. 2001). It is tempting, however, to speculate that this low angular momentum material might actually build a (near-zero angular momentum) bulge component (cf. van den Bosch 1998; Kepner 1999). The mass of such a bulge depends on the details of the angular momentum exchange during cooling. The mass with negative specific angular momentum may combine with very little mass but with high positive specific angular momentum (yielding a minimum bulge mass), or with a relatively large amount of mass with small, but positive, specific angular momentum (yielding a maximum bulge mass). As a simple intermediate case, consider the scenario in which the zero angular momentum bulge forms out of the material with AMD

$$
p_{\text {bulge }}(l)= \begin{cases}p(l) & l<0 \\ p(-l) & l \geq 0\end{cases}
$$

If the remaining material builds a disk, the resulting bulge-to-disk mass ratio is

$$
B / D=\frac{2 f_{\text {gas }}}{1-2 f_{\text {gas }}}
$$

while the angular momentum distribution of the disk material is given by $p_{\text {disk }}(l) \equiv p(l)-$ $p(-l)$ where $l \geq 0$. Under the standard assumption that this material conserves its specific angular momentum, and that the dark matter follows an NFW density profile (Navarro, Frenk \& White 1997), the surface density $\Sigma(R)$ of the resulting disk component can be computed using the relation

$$
\frac{M_{\text {disk }}}{1-2 f_{\text {gas }}} \int_{l_{1}}^{l_{2}} p_{\text {disk }}(l) \mathrm{d} l=2 \pi \int_{R_{1}}^{R_{2}} \Sigma(R) R \mathrm{~d} R
$$

where $l$ and $R$ are related according to

$$
l=\frac{R V_{c}(R)}{R_{\mathrm{vir}} V_{\mathrm{vir}}}=\sqrt{x \frac{\ln (1+c x)-c x /(1+c x)}{\ln (1+c)-c /(1+c)}}
$$


with $x=R / R_{\mathrm{vir}}, V_{c}(R)$ the circular velocity at radius $R$, and $c$ the NFW concentration parameter for which we adopt $c=10$ throughout. For simplicity we ignore the self-gravity of the disk, so that $V_{c}(R)$ reflects the circular velocity of the NFW halo.

In Figure 10 we plot the resulting disk surface density distributions $\Sigma(R)$ for four representative halos from our sample of 23 (thick solid lines). In each panel we indicate the bulge-to-disk ratio computed with equation (11). The thin lines correspond to disks that would form out of an angular momentum distribution given by

$$
p(l)=\frac{\zeta \mu(\mu-1)}{(\zeta l+\mu-1)^{2}}
$$

which corresponds to the 'Universal' profile of equation 9. Here

$$
\zeta=\frac{1}{\sqrt{2} \lambda_{\text {gas }}}\left(1-\mu\left[1-(\mu-1) \ln \left(\frac{\mu}{\mu-1}\right)\right]\right) .
$$

where $\lambda_{\text {gas }}$ is the spin parameter of the gas in the halo under consideration (listed in Table 1). We adopt $\mu=1.25$, which corresponds to the median value of all halos analyzed by B01.

Whereas the disks forming out of the AMDs of the gas analyzed here have surface brightness profiles that are very close to exponential (corresponding to a straight line in the panels on the left), this is not the case for disks forming out of the 'Universal' AMD. The largest difference between the two surface brightness profiles is apparent at small radii. This is due to the bulge-formation scenario included in one case but not the other. In fact, as shown by van den Bosch (2001), if a simple bulge formation scenario is included, the 'Universal' AMD also yields near-exponential disks. One problem that is not solved by turning low angular momentum material into a bulge, however, is the fact that the 'Universal' AMD yields disks with a clear truncation radius which occurs at too high surface density (van den Bosch 2001). However, the disks forming out of the AMDs of the gas analyzed here continue as exponentials to much lower surface brightness, suggesting that the cell averaging adopted by B01 underestimated the true $j_{\max }$.

Thus, the angular momentum distributions of the gas in proto-galaxies seem consistent with the observed surface density distributions of disk galaxies if the material with negative specific angular momentum is assumed to make a bulge component. Nevertheless, several problems remain.

First of all, since $0.05 \lesssim f_{\text {gas }} \lesssim 0.5$ in the scenario considered above, a large fraction of the halos will form bulge dominated 'early-type' systems. If we use a bulge-to-disk ratio of $B / D=1$ to discriminate between late and early-type galaxies, disk dominated late type galaxies only form in $\sim 60$ percent of all halos in our sample. Furthermore, with a minimum 
$f_{\text {gas }}$ of about 5 percent (see Figure 2), no systems will form with $B / D<0.1$, so the question of how bulge-less dwarf and LSB galaxies form (van den Bosch 2001; van den Bosch et al. 2001) is unanswered.

Another problem concerns the various misalignments of angular momentum. As we have shown in the lower panels of Figure 7, not all gas has the same direction of angular momentum. When the self-gravity of the gas becomes important, gas parcels with different angular momentum directions (moving on non-coplanar circular orbits) will exert torques on each other, resulting in a redistribution of angular momentum. In addition, when the gas is misaligned with the potential of the dark matter halo ${ }^{4}$, transfer of angular momentum between the gas and dark matter will occur. If the gas predominantly transfers angular momentum to the dark matter, which one might naively expect since the latter is the dominant mass component, this results in an additional problem as many authors in the past have pointed out: In order to explain the observed distribution of disk scale lengths the gas cannot lose much of its angular momentum.

\section{Conclusions}

It is generally assumed that the gas and the dark matter in a proto-galaxy have the same distribution of specific angular momentum. This is motivated by the fact that both mass components experience the same tidal torques. However, the two components undergo rather different relaxation mechanisms: whereas the dark matter undergoes collisionless, violent relaxation, the gas gets shocked which could in principle cause a redistribution of its angular momentum distribution. In this paper we have used numerical simulations to test, for the first time, whether the ansatz that gas and dark matter have similar initial angular momentum distributions is correct. We presented a numerical simulation of structure formation in a $\Lambda \mathrm{CDM}$ cosmology including both dark matter and a non-radiative gas. For $\sim 380$ halos with virial masses in the range $4.8 \times 10^{9} h^{-1} \mathrm{M}_{\odot} \leq M_{\mathrm{vir}} \leq 1.1 \times 10^{12} h^{-1} \mathrm{M}_{\odot}($ at $z=3$ ) we computed the angular momenta of both the gas and the dark matter.

We have shown that on average the gas and dark matter have the same distribution of spin parameters and that their detailed angular momentum distributions in individual halos are remarkably similar. Evidently, shocking during the virialization process does not decouple

\footnotetext{
${ }^{4}$ We emphasize here that any possible misalignment between the gas and the moment of inertia of the dark matter halo is not related to the misalignment angle $\theta$ between the angular momentum vectors of the gas and dark matter: halos are not rotationally supported systems, and as shown by Porciani, Dekel \& Hoffman (2002), the spin and inertia of dark matter halos are poorly aligned.
} 
the angular momentum of the gas from that of the dark matter, supporting the standard assumption that gas and dark matter have identical angular momentum distributions. We have shown that these results are robust by also analyzing a high resolution numerical simulation of a single cluster-sized halo at $z=0$ in a much larger cosmological volume.

A careful analysis of the detailed angular momentum distributions reveals that they are inconsistent with the 'Universal' profile suggested by B01. In the angular momentum distributions presented here between 5 and 50 percent of the mass has negative specific angular momentum, whereas B01 have (implicitly) assumed that all mass has positive specific angular momentum. Furthermore, our angular momentum distributions have wings out to very high specific angular momentum, whereas the profile suggested by B01 has a cut-off at a certain $j_{\max }$ that results in disk truncation at too high surface density (van den Bosch 2001).

The fact that relatively large mass fractions have negative specific angular momentum suggests that the standard assumption of detailed specific angular momentum conservation, which is often made in theories of disk formation, cannot be correct. After all, disk galaxies do not typically contain material with negative specific angular momentum. We have suggested that during the cooling process the gas with negative specific angular momentum may collide/shock with material with positive specific angular momentum to build a bulge component with zero angular momentum. In such a picture at least the total amount of angular momentum of the gas is conserved, and we have shown that it can produce disks with near-exponential surface density distributions without the small truncation radii implied by the 'Universal' profile of B01. Numerical simulations of disk formation that include cooling do indeed seem to reveal the formation of a dense central knot of gas in addition to the disk (e.g., Katz \& Gunn 1991; Navarro \& White 1994). Although this 'bulge' is typically interpreted as forming out of sub-clumps that lose their specific angular momentum to the dark matter through dynamical friction, our results suggest that even without substructure a bulge may form naturally. In fact, taking the results at face value suggests that $\sim 40$ percent of all halos are unable to form disk dominated galaxies, simply because they have too large mass fractions with negative specific angular momentum. In addition, systems with bulge-to-disk mass ratios below 10 percent will be extremely rare.

In reality the formation of disk galaxies will be more complicated as depicted above. We have shown that the angular momentum vectors of the gas and dark matter are misaligned by on average $\sim 35^{\circ}$. Surprisingly the amount of misalignment is not a measure for the degree to which the respective angular momentum distributions agree or disagree with each other. There is a weak tendency for the misalignment to be weaker in halos that are more massive or have larger spin parameters. Higher resolution simulations are required 
to investigate the amount and origin of this misalignment in more detail. In addition to this misalignment between the two components, the angular momentum vector of each individual component can change direction quite dramatically with radius. This is typically interpreted as originating from decoherence in the angular momentum orientation of material accreted at different times (Ryden 1988; Quinn \& Binney 1992) and is often invoked as a mechanism to create warps in disk galaxies (e.g., Ostriker \& Binney 1989). Although the exact implications of these various misalignments are currently unclear, and require a more detailed investigation, it is clear that any misalignment of the gas with either itself or with the inertia moment of the dark matter halo has to vanish if one wants to build a stable disk. If this results in a significant transfer of angular momentum from the gas to the dark matter these misalignments might be an additional cause for the angular momentum catastrophe hampering the formation of sufficiently extended disk galaxies in numerical simulations.

Finally, we emphasize that the implications for disk/bulge formation discussed above are highly speculative. In our simulations the gas was not allowed to cool, and we used the angular momentum distributions of the gas found at $z=3$ to speculate about the fate of the gas once cooling were turned on. In reality the gas will already have (partially) cooled inside sub-clumps that merge to form the systems analyzed here, and this will also impact on the angular momentum distribution of the gas and its subsequent evolution (e.g., dynamical friction). On the other hand, it has been argued by several authors that in order to prevent the angular momentum catastrophe the gas has to be prevented from cooling inside sub-clumps for instance by feedback processes or preheating (e.g., Navarro \& White 1994; Weil, Eke \& Efstathiou 1998; Sommer-Larsen, Gelato \& Vedel 1999). Under those circumstances the angular momentum distribution of the gas may in fact resemble those analyzed here, although it is unclear to what extent these heating processes influence the angular momentum distributions of the gas prior to being incorporated in the halos. For instance, processes related to feedback and re-ionization create pressure gradients in the gas which may decouple the angular momentum distribution of the gas from that of the dark matter. In a follow-up paper (Abel et al. 2002) we address in more detail the impact such pressure forces may have on the angular momentum distributions in proto-galaxies.

We are grateful to Naoki Yoshida for providing his high resolution numerical simulation, to James Bullock, Andreas Burkert, Avishai Dekel, Ariyeh Maller, Joel Primack and Risa Wechsler for stimulating discussions, and to Volker Springel for his help with the simulations. This work has partially been supported through NSF grants ACI96-19019 and AST-9803137. We also acknowledge support from the Grand Challenge Cosmology Consortium, and from the EC RTN network "The Physics of the Intergalactic Medium". 


\section{REFERENCES}

Abel, T., van den Bosch, F. C., Croft, R. C., Hernquist, L., \& White, S. D. M. 2002, in preparation

Avila-Reese, V., \& Firmani, C. 2000, RevMexAA, 36, 23

Barnes, J., \& Efstathiou, G. 1987, ApJ, 319, 575

Bryan, G., \& Norman, M. 1998, ApJ, 495, 80

Buchalter, A., Jimenez, R., \& Kamionkowski, M. 2001, MNRAS, 322, 43

Bullock, J. S., Dekel, A., Kolatt, T. S., Kravtsov, A. V., Klypin, A. A., Porciani, C., \& Primack, J. R. 2001, ApJ, 555, 240 (B01)

Bullock, J. S., Kravtsov, A. V., \& Colín, P. 2002, ApJ, 564, L1

Catelan, P., \& Theuns, T. 1996, MNRAS, 282, 436

Crittenden, R. G., Natarajan, P., Pen, U. L., \& Theuns, T. 2001, ApJ, 559, 552

Croft, R. A. C., \& Metzler, C .A. 2000, ApJ, 545, 561

Dalcanton, J. J., Spergel, D. N., \& Summers, F. J. 1997, ApJ, 482, 659

Davis, M., Efstathiou, G., Frenk, C. S., \& White, S. D. M. 1985, ApJ, 292, 371

de Jong, R.S., \& Lacey, C. 2000, ApJ, 545, 781

Doroshkevich, A. G. 1970, Astrofizika, 6, 581

Efstathiou, G., Bond, J. R., \& White, S. D. M. 1992, MNRAS, 258, 1

Eisenstein, D. J., \& Hut, P. 1998, ApJ, 496, 605

Fall, S. M., \& Efstathiou, G. 1980, MNRAS, 193, 189

Firmani, C., \& Avila-Reese, V. 2000, MNRAS, 315, 457

Heavens, A. F., \& Jimenez, R. 1999, MNRAS, 305, 770

Heavens, A. F., Refegier, A., \& Heymans, C. 2000, MNRAS, 319, 649

Hoyle, F. 1953, ApJ, 118, 513

Jimenez, R., Padoan, P., Matteucci, F., \& Heavens, A.F. 1998, MNRAS, 296, 1089 
Katz, N., \& Gunn, J. E. 1991, ApJ, 377, 365

Kauffmann, G., Colberg, J. M., Diaferio, A., \& White, S. D. M. 1999, MNRAS, 303, 188

Kepner, J. V. 1999, ApJ,520, 59

Knebe, A., Devriendt, J., Mahmood, A., \& Silk, J. 2002, MNRAS, 329, 813

Lee, J., \& Pen, U. L. 2000, ApJ, 532, L5

Lee, J., \& Pen, U. L. 2001, ApJ, 555, 106

Maller, A. H., Dekel, A., \& Somerville, R. S. 2001, MNRAS, 329, 423

Maller, A. H., Dekel, A. 2002, preprint (astro-ph/0201187)

McKay, T. A., et al. 2001, preprint (astro-ph/0108013)

Mestel, L. 1963, MNRAS, 126, 553

Mo, H. J., Mao, S., \& White, S. D. M. 1998, MNRAS, 295, 319

Natarajan, P. 1999, ApJ, 512, 105

Natarajan, P., Crittenden, R. G., Pen, U. L., \& Theuns, T. 2001, PASA, 18, 198

Navarro, J. F., \& Benz, W. 1991, ApJ, 380, 320

Navarro, J. F., \& White, S. D. M. 1994, MNRAS, 267, 401

Navarro, J. F., Frenk, C. S., \& White, S. D. M. 1997, ApJ, 490, 493

Navarro, J. F., \& Steinmetz, M. 2000, ApJ, 538, 477

Ostriker, E. C., \& Binney, J. J. 1989, MNRAS, 237, 785

Peebles, P. J. E. 1969, ApJ, 155, 393

Porciani, C., Dekel, A., \& Hoffman, Y. 2002, MNRAS, 332, 325

Quinn, T., \& Binney, J. J. 1992, MNRAS, 255, 729

Ryden, B. S. 1988, ApJ, 329, 589

Sommer-Larsen, J., Gelato, S., \& Vedel, H. 1999, ApJ, 519, 501

Springel, V. 1999, PhD Thesis, Ludwig-Maximilians University 
Springel, V., White, S. D. M., Tormen, G., \& Kauffmann, G. 2000, MNRAS, 328, 726

Springel, V., Yoshida, N., \& White, S. D. M. 2001, New Astronomy, 6, 79

Steinmetz, M., \& Navarro, J. F. 1999, ApJ, 513, 555

Thacker, R. J., \& Couchman, H. M. P. 2001, ApJ, 555, L17

Tormen, G., Bouchet, F. R., \& White, S. D. M. 1997, MNRAS, 286, 865

van den Bosch, F. C. 1998, ApJ, 507, 601

van den Bosch, F. C. 2000, ApJ, 530, 177

van den Bosch, F. C. 2001, MNRAS, 327, 1334

van den Bosch, F. C. 2002, MNRAS, 332, 456

van den Bosch, F. C., Burkert, A., \& Swaters, R. A. 2001, MNRAS, 326, 1205

van Waerbecke, L., et al. 2000, A\&A, 358, 30

Warren, M. S., Quinn, P. J., Salmon, J. K., \& Zurek, W. H. 1992, ApJ, 399, 405

Weil, M. L., Eke, V. R., \& Efstathiou, G. 1998, MNRAS, 300, 773

White, S. D. M. 1984, MNRAS, 286, 38

White, S. D. M., \& Rees, M. J. 1978, MNRAS, 183, 341

Yoshida, N., Springel, V., White, S. D. M., \& Tormen, G. 2000a, ApJ, 535, L103

Yoshida, N., Springel, V., White, S. D. M., \& Tormen, G. 2000b, ApJ, 544, L87 

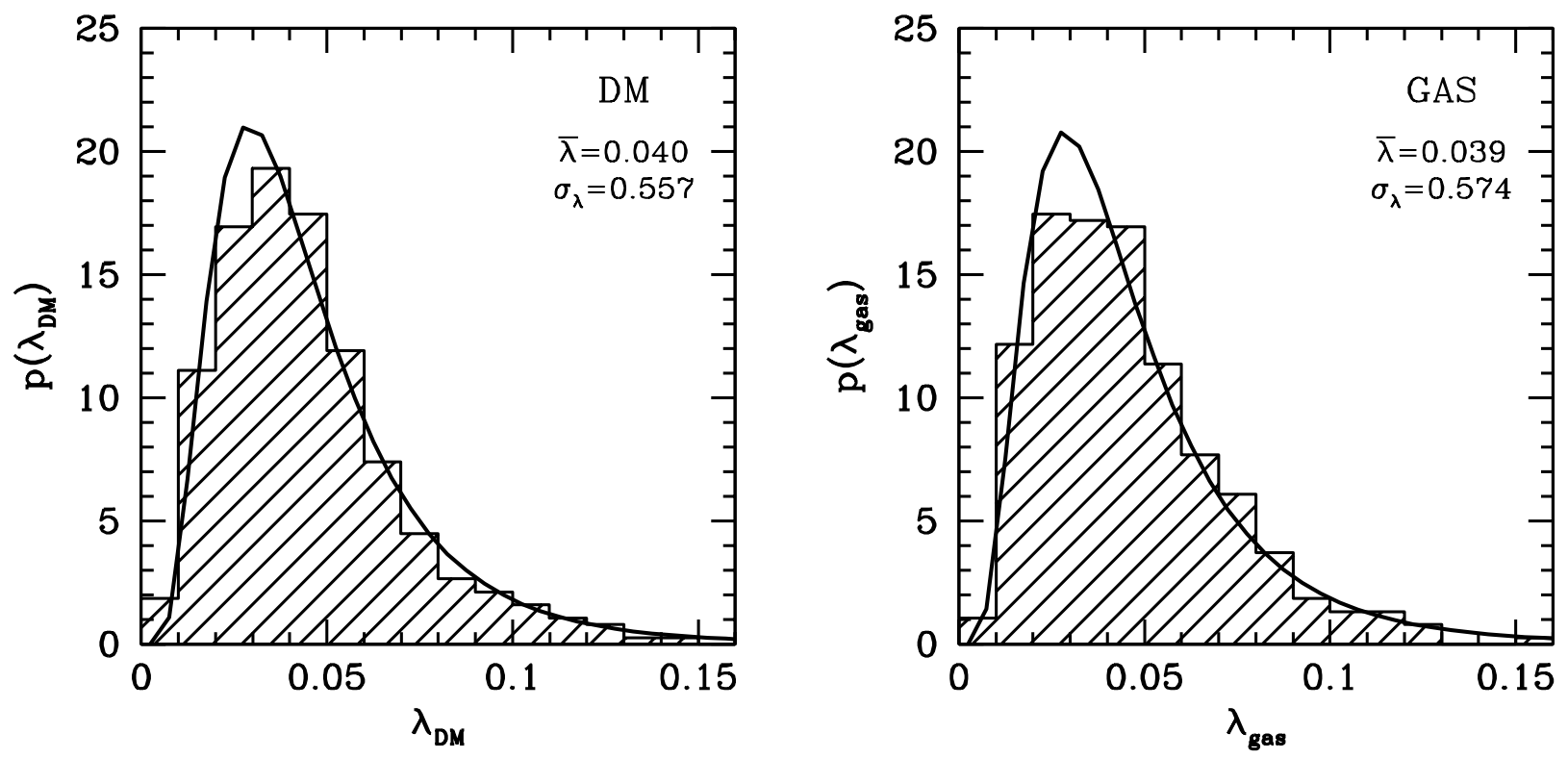

Fig. 1. - The distributions of $\lambda_{\mathrm{DM}}$ (right panel) and $\lambda_{\text {gas }}$ (left panel) for all 378 halos in our sample. Solid lines are the best fit log-normal distributions of equation (6). The values of the best fit parameters are indicated in each panel. Note that the gas and dark matter have very similar distributions of spin parameters. 

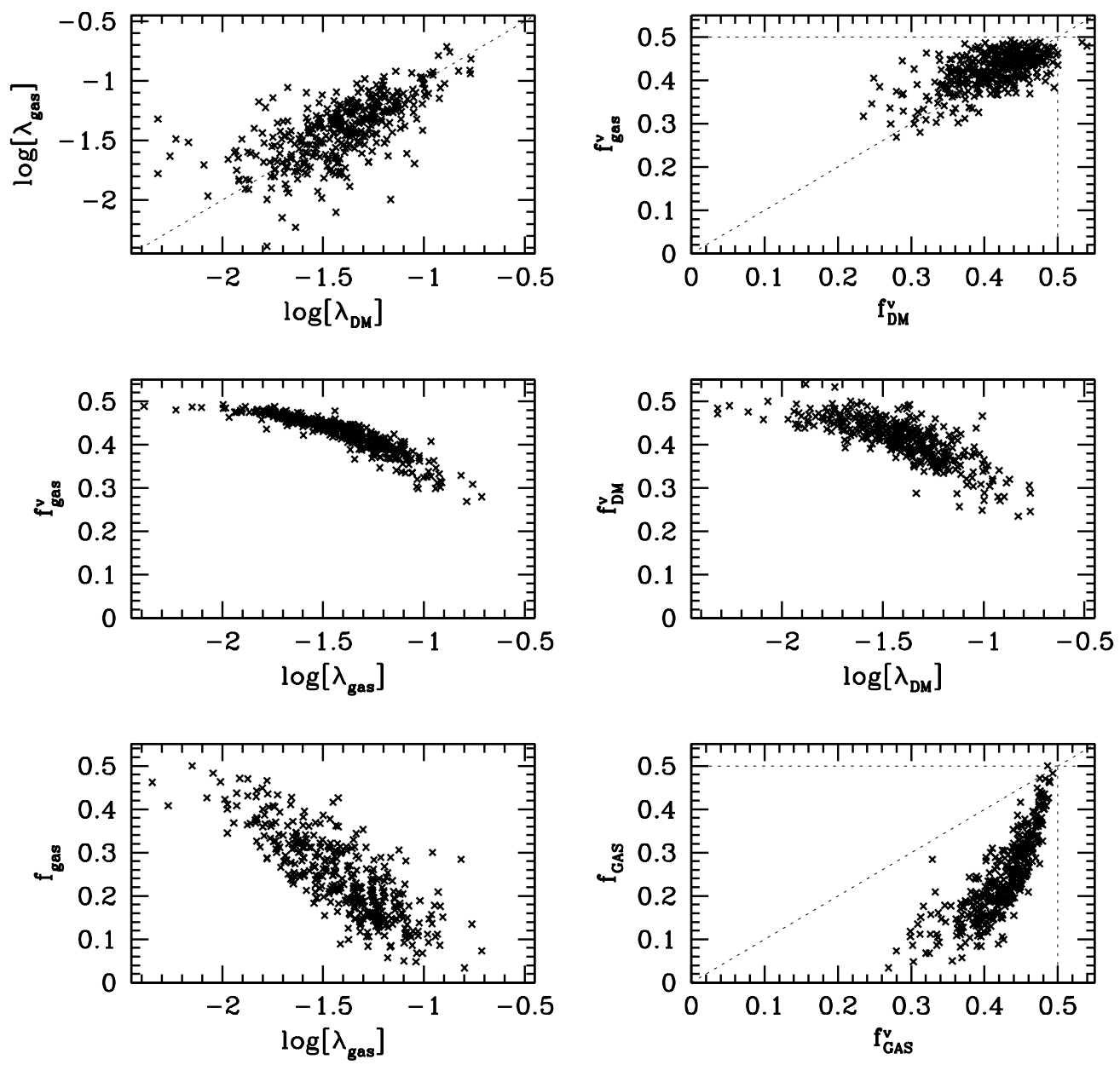

Fig. 2.- A comparison of various global angular momentum parameters of the dark matter and gas for all 378 halos in our sample. The upper left panel plots $\lambda_{\text {gas }}$ versus $\lambda_{\mathrm{DM}}$. The dotted line corresponds to perfect agreement (i.e., $\lambda_{\text {gas }}=\lambda_{\mathrm{DM}}$ ) and is plotted for comparison. Although on average the spin parameters of gas and dark matter are similar, for each individual halo the difference between the spin parameters of both components can be relatively large. In the upper right panel we plot $f_{\text {gas }}^{v}$ versus $f_{\mathrm{DM}}^{v}$. Again, on average the mass fractions with negative specific angular momentum are similar for the gas and the dark matter (i.e., $\left.\left\langle f_{\mathrm{DM}}^{v} / f_{\text {gas }}^{v}\right\rangle=0.97 \pm 0.10\right)$. The two panels in the middle plot $f_{\text {gas }}^{v}$ versus $\lambda_{\text {gas }}$ (left) and $f_{\mathrm{DM}}^{v}$ versus $\lambda_{\mathrm{DM}}$ (right). Note how the total specific angular momentum of each component is anti-correlated with its respective mass fraction with negative $j_{z}$. The same is true when plotting $f_{\text {gas }}$ rather than $f_{\text {gas }}^{v}$ (lower left panel). Finally, the lower right panel plots $f_{\text {gas }}$ versus $f_{\text {gas }}^{v}$. As expected, all halos have $f_{\text {gas }}<f_{\text {gas }}^{v}$. Note however that the difference depends on $f_{\text {gas }}^{v}$ and thus on $\lambda_{\text {gas }}$. See the text for a more detailed discussion. 


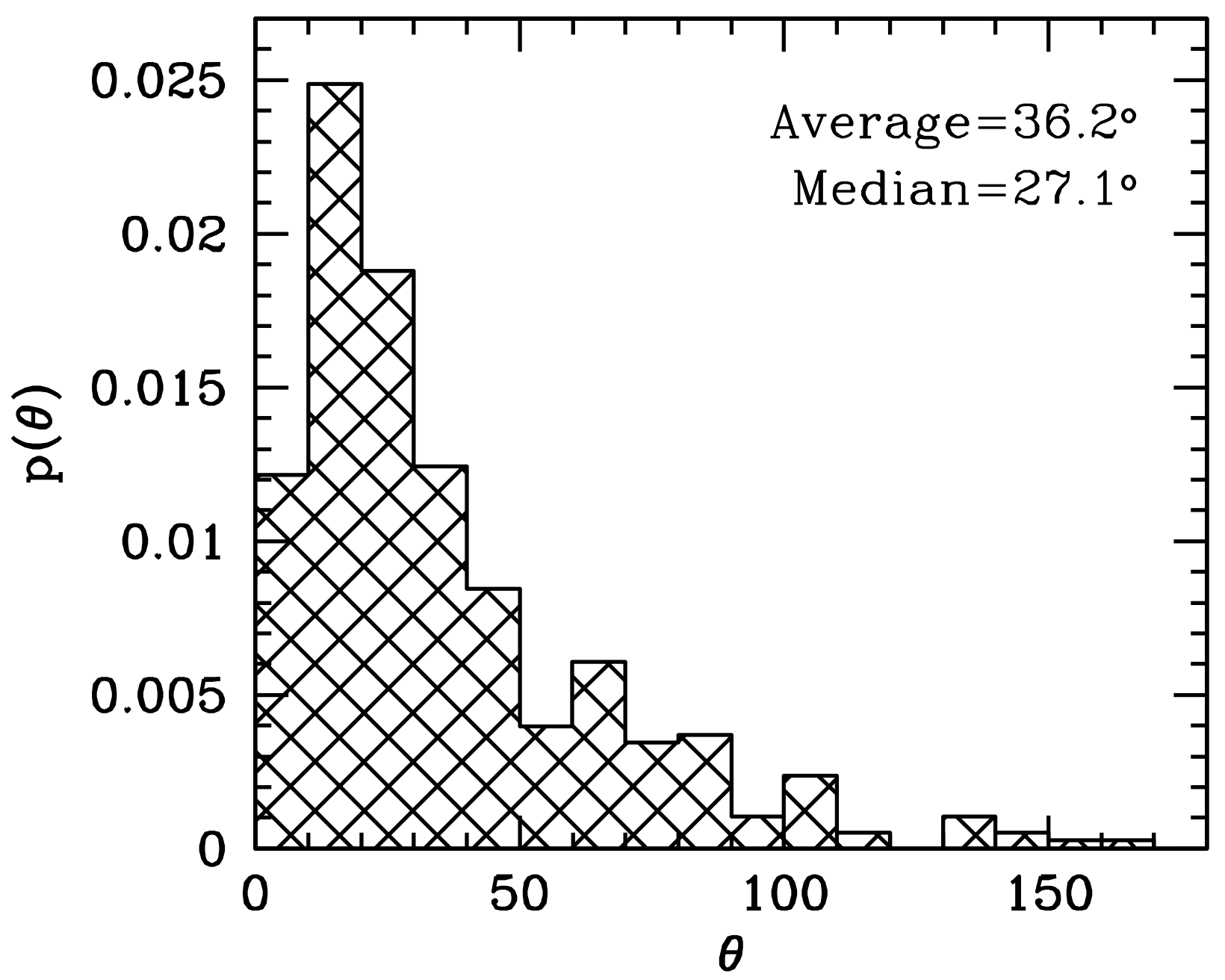

Fig. 3.- The distribution of the angle $\theta$ between the total angular momentum vectors of the dark matter and the gas. The average and mean of the distribution are indicated. 

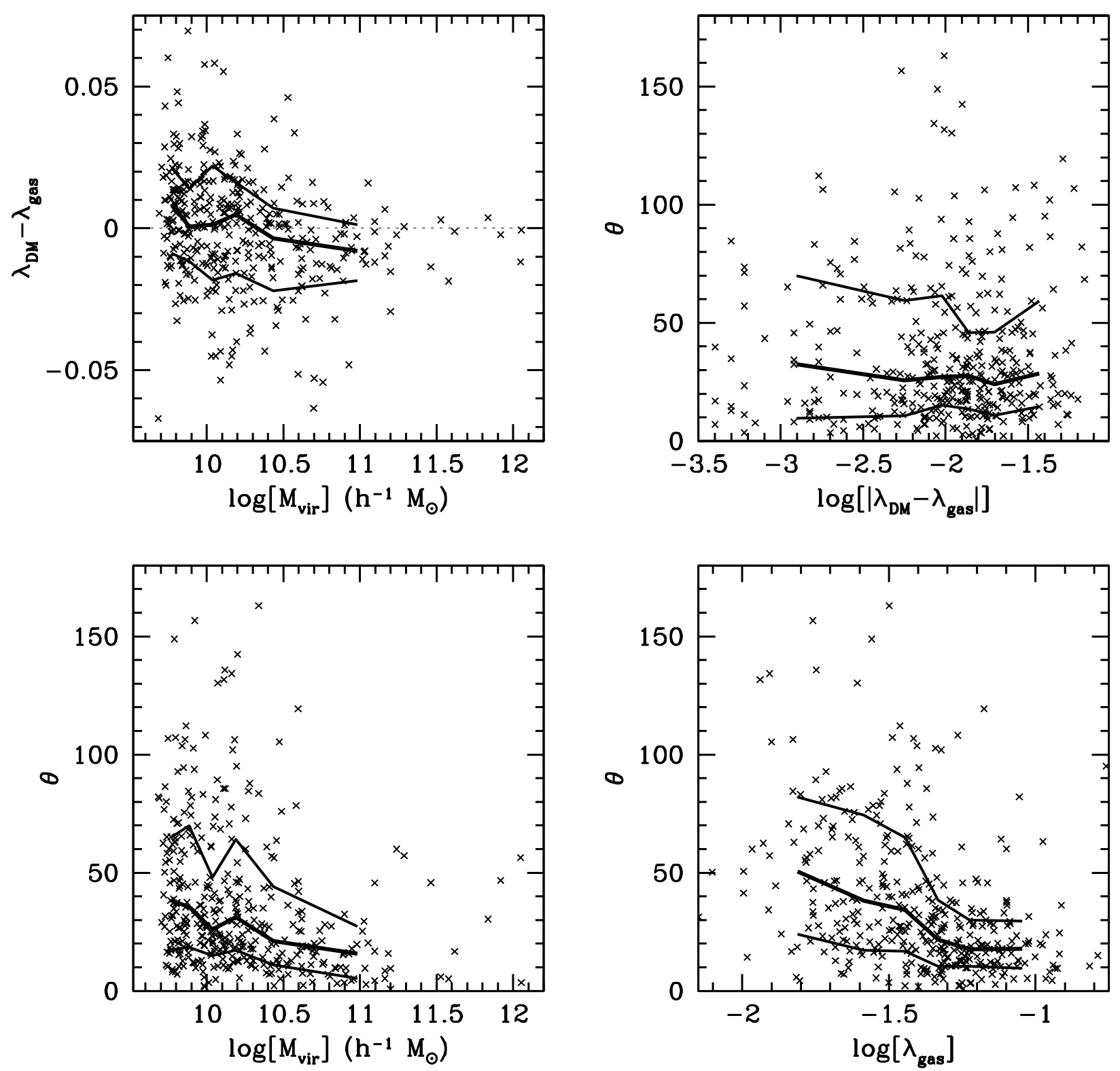

Fig. 4.- The upper left panel plots $\lambda_{\mathrm{DM}}-\lambda_{\text {gas }}$ as function of the halo virial mass. The thick solid line in the middle indicates the run of the average $\lambda_{\mathrm{DM}}-\lambda_{\text {gas }}$, while the upper and lower solid lines indicate the 68 percent interval of $\lambda_{\mathrm{DM}}-\lambda_{\text {gas }}$ at fixed $M_{\mathrm{vir}}$. Note that the difference in the spin parameters of the gas and the dark matter do not depend significantly on the halo virial mass. The other panels plot $\theta$ as function of the absolute difference between the spin parameters of gas and dark matter (upper right panel), the total virial mass (lower left panel), and the spin parameter of the gas (lower right panel). Thick solid lines correspond to the mean and 68 percent interval of $\theta$. Whereas there is no dependence of $\theta$ on $\left|\lambda_{\mathrm{DM}}-\lambda_{\text {gas }}\right|$, the average misalignment between the angular momentum vectors of the gas and dark matter is larger in less massive halos and in halos with smaller spin parameters. 


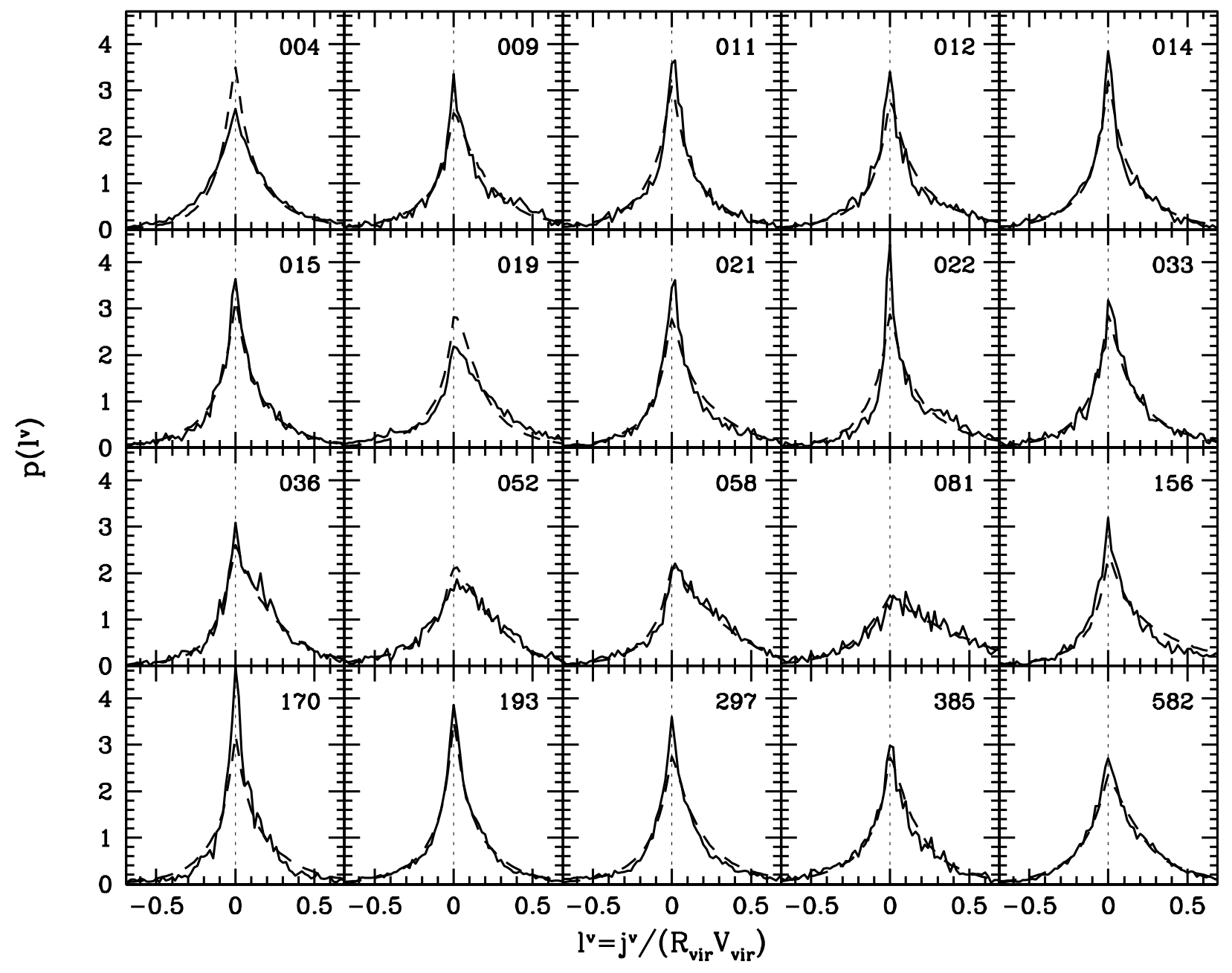

Fig. 5.- The angular momentum distributions $p\left(l^{v}\right)$ for the 20 halos listed in Table 1. Solid and dashed lines correspond to the dark matter and gas, respectively. Note the remarkable similarity between the angular momentum distributions of the gas and the dark matter. 


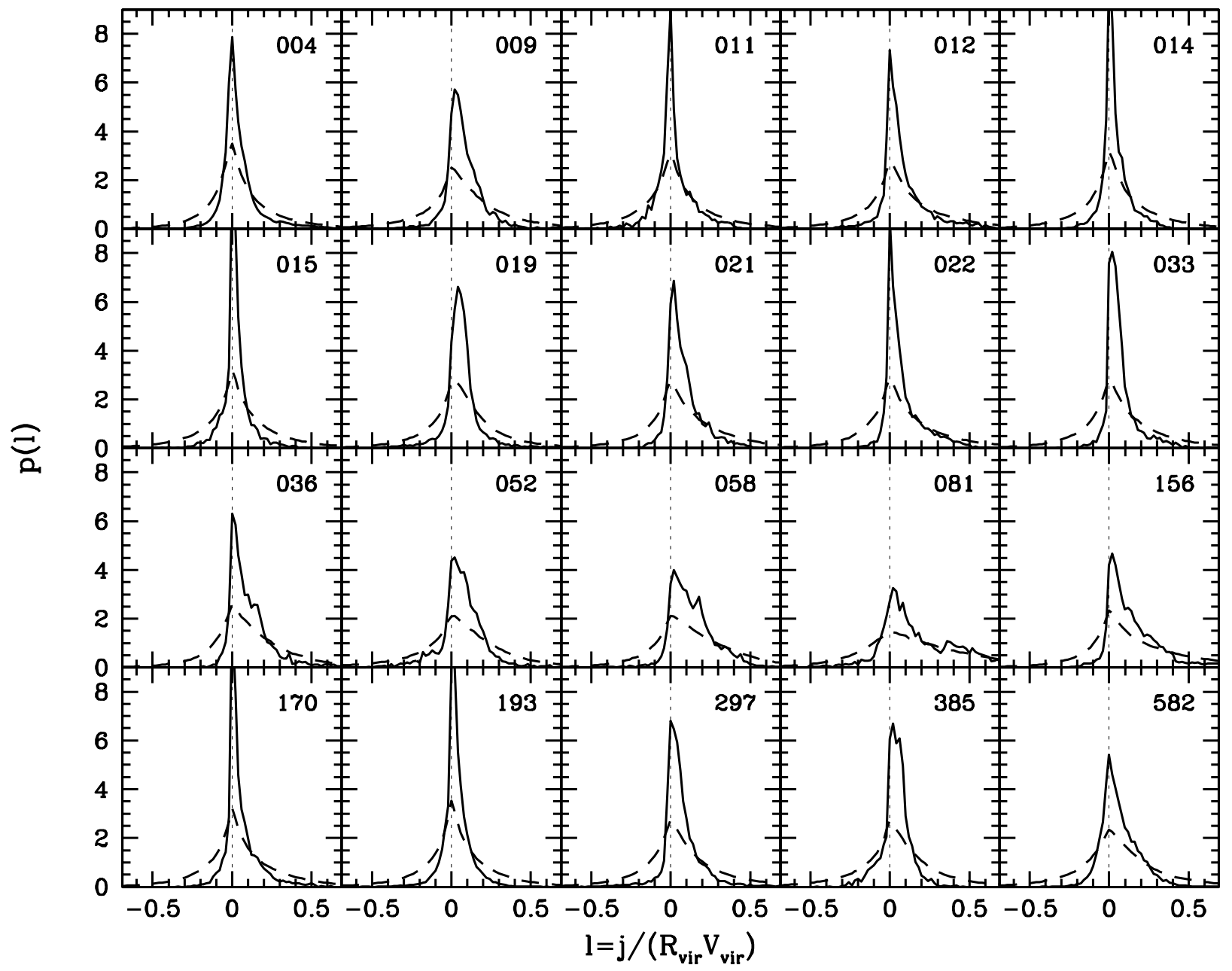

Fig. 6.- A comparison of the angular momentum distributions $p(l)$ (solid lines) and $p\left(l^{v}\right)$ (dashed lines) of the gas in the 20 halos listed in Table 1 . Note how $p(l)$ typically has much smaller wings at negative specific angular momentum than $p\left(l^{v}\right)$ (cf, lower right panel of Figure 2). 


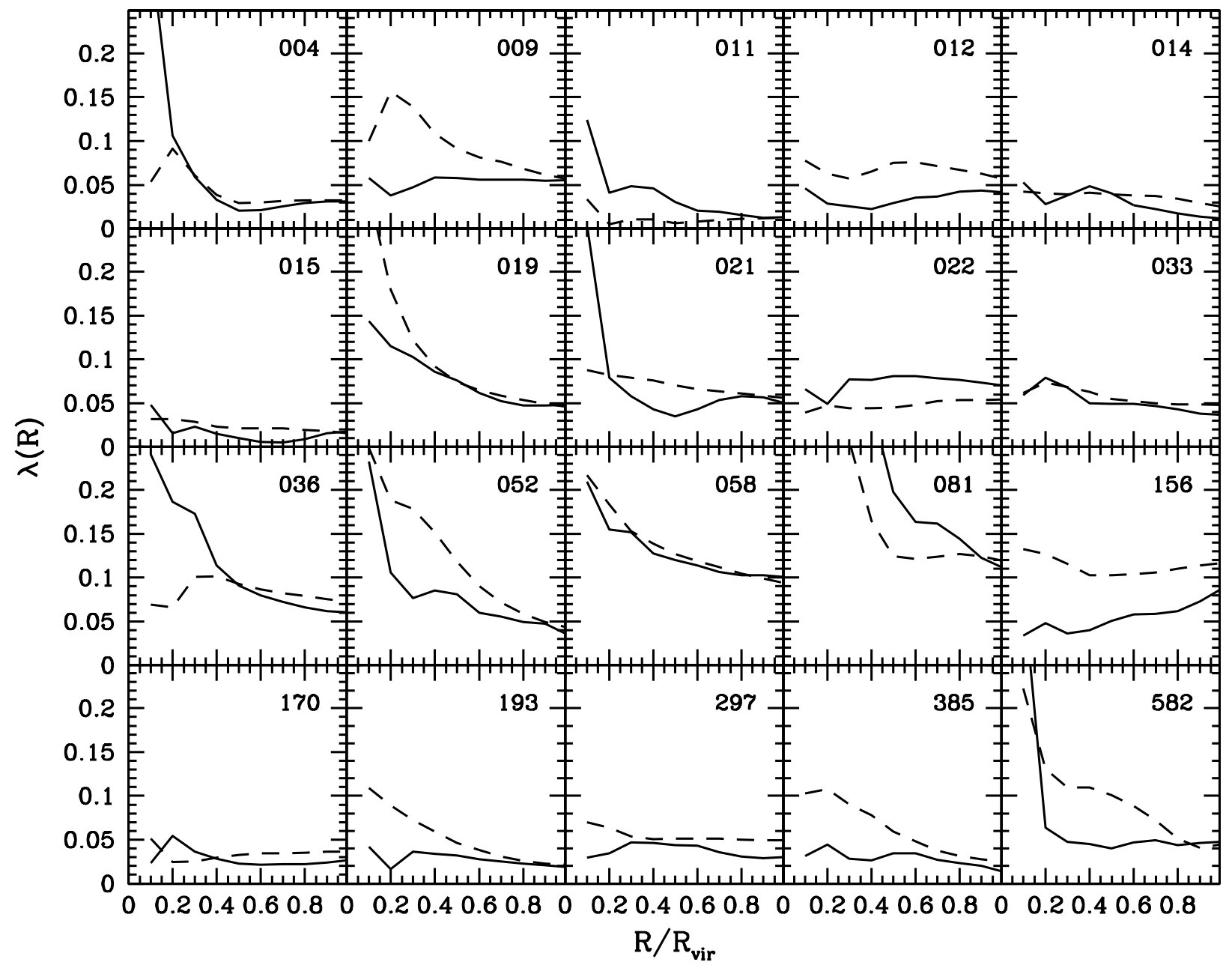

Fig. 7.- The values of the spin parameter inside radius $R$ as functions of $R / R_{\text {vir }}$ for the 20 halos listed in Table 1. Solid (dashed) lines correspond to the dark matter (gas). Typically $\lambda(R)$ either decreases with radius or stays roughly constant. Although in some halos $\lambda_{\mathrm{DM}}(R)$ and $\lambda_{\text {gas }}(R)$ can be quite different, there is no indication for a systematic difference between $\lambda(R)$ of the gas and dark matter. 


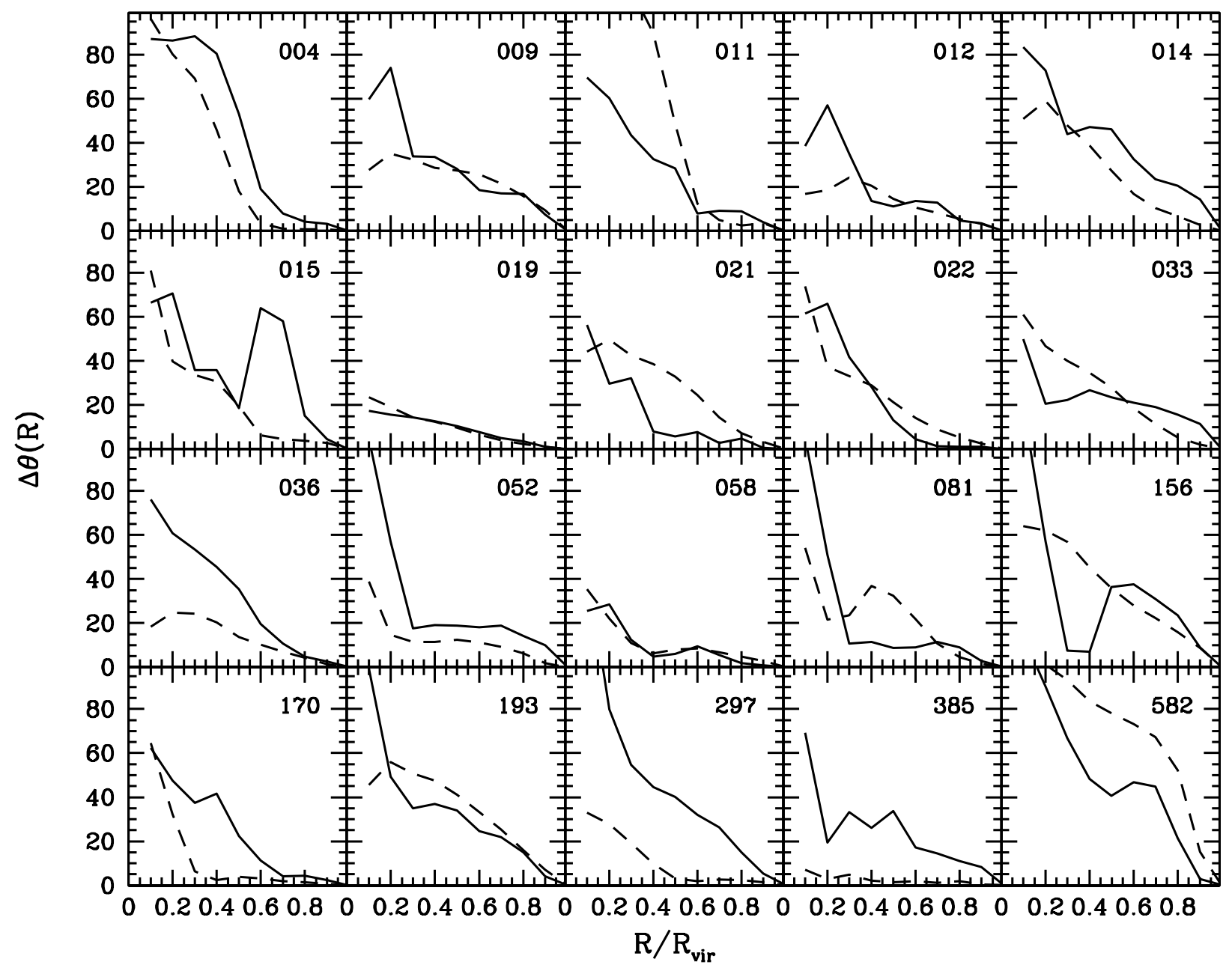

Fig. 8.- The misalignment angle $\Delta \theta(R)$ between the angular momentum vector inside radius $R$ and that of the entire halo. As in Figure 7 solid (dashed) lines correspond to the dark matter (gas). Results are plotted as function of $R / R_{\mathrm{vir}}$. Note that the angular momentum directions of both the gas and dark matter can vary quite significantly with radius, and in some cases is clearly different for the gas and the dark matter. 

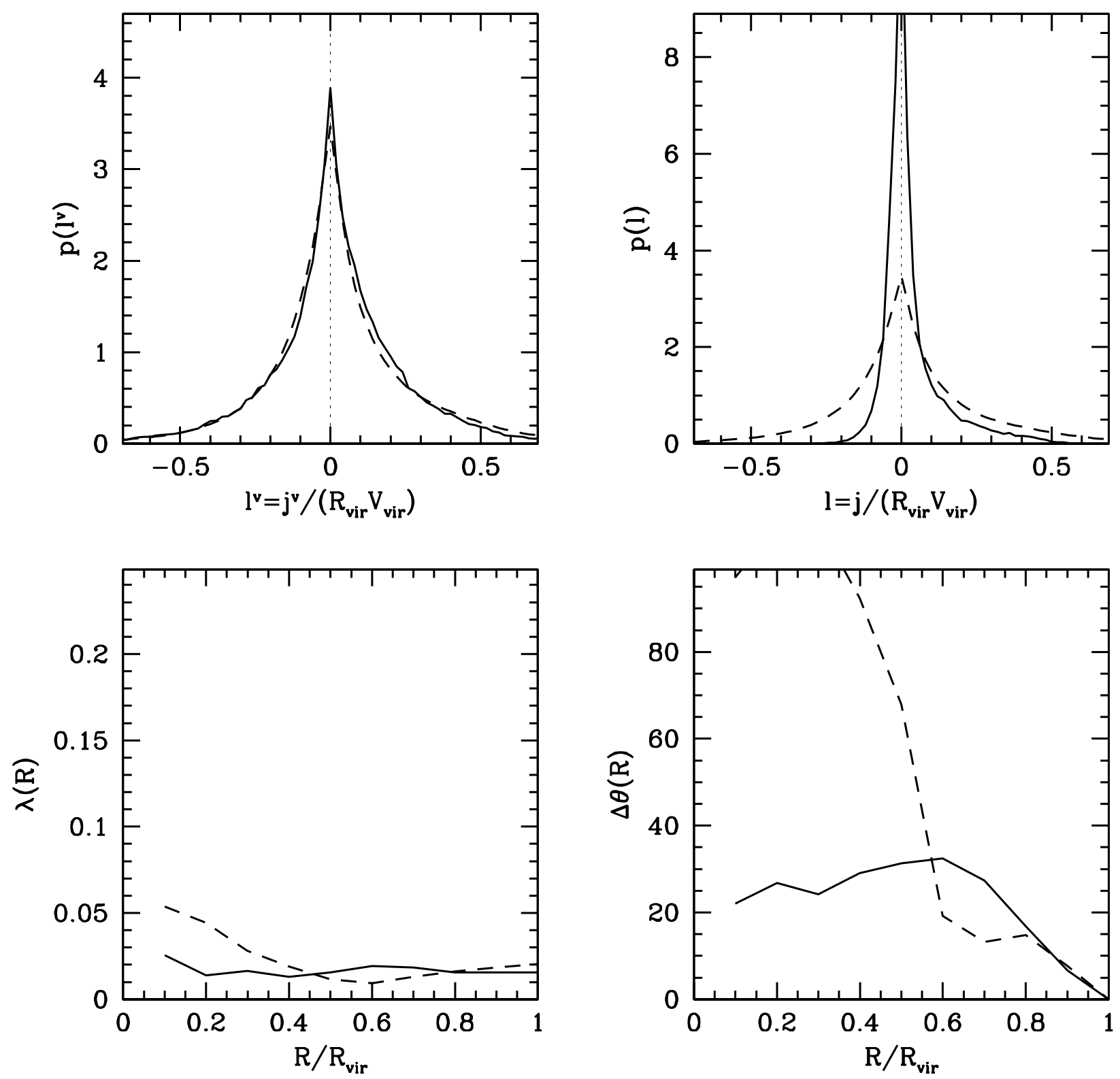

Fig. 9.- Results at $z=0$ for the high resolution simulation of cluster 'S0' described in Section 3.3. The upper left panel plots $p\left(l^{v}\right)$ for both the dark matter (solid line) and the gas (dashed line). As for the halos presented in Figure 5 the dark matter and gas have virtually indistinguishable distributions of specific angular momentum. The upper right panel compares $p(l)$ (solid line) with $p\left(l^{v}\right)$ for the gas (cf. Figure 6). Note that even when considering the streaming motions of the gas, there is a large fraction of gas mass with negative specific angular momentum (see also Table 1 ). The lower two panels plot $\lambda(R)$ and $\Delta \theta(R)$ for halo 'S0', where the solid (dashed) lines correspond to the dark matter (gas). A comparison with Figures 7 and 8 shows that halo 'S0' behaves similar as the halos analyzed in Section 3.2. 

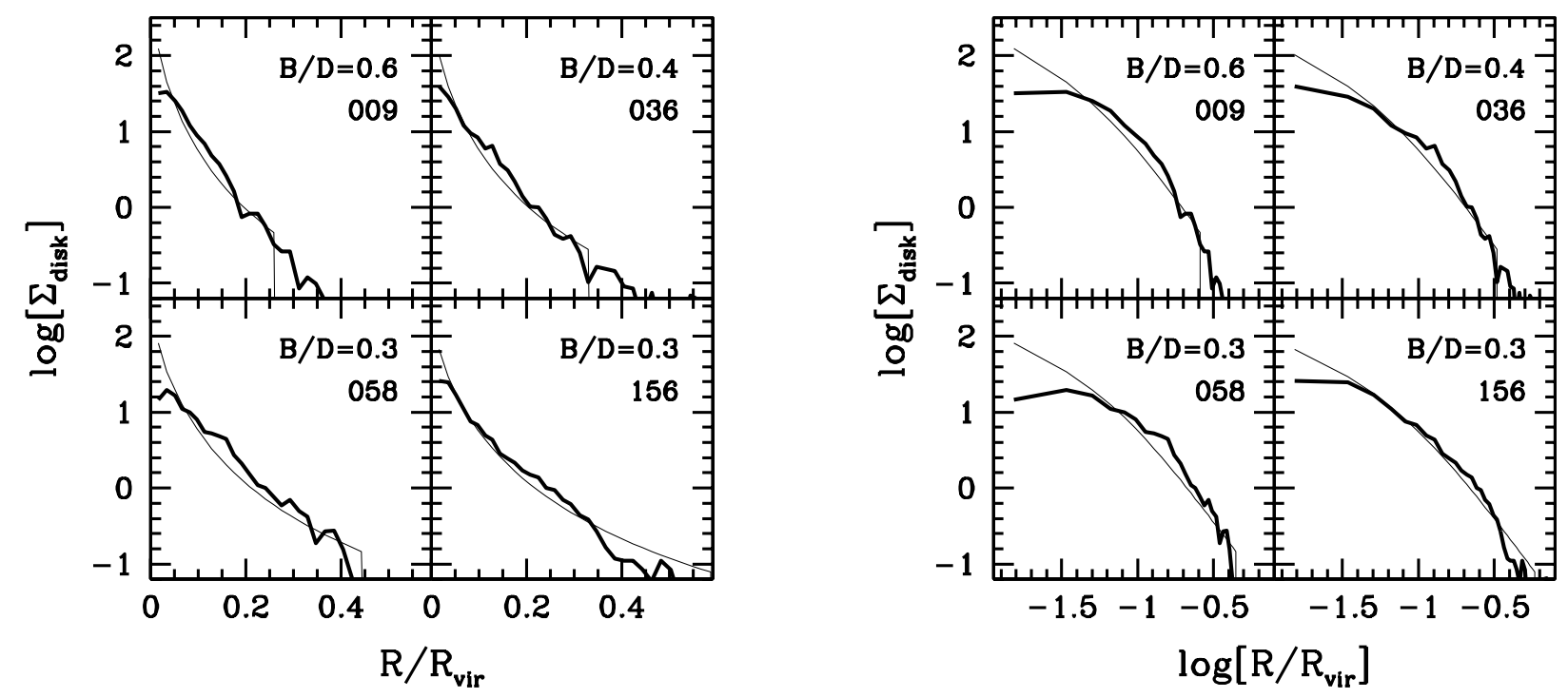

Fig. 10.- The thick solid lines correspond to the density distributions (in arbitrary units) of the disks that form in four halos listed in Table 1 under the assumptions that (i) material with negative specific angular momentum forms a zero-angular momentum bulge together with material with the same, but positive, specific angular momentum distribution (e.g., equation [10]), (ii) the remaining material forms a disk under detailed angular momentum conservation, (iii) the dark matter halo has an NFW density distribution with $c=10$, and (iv) the self-gravity of the disk can be ignored. The resulting bulge-to-disk mass ratios $B / D$ are indicated in each panel. The thin solid lines correspond to disks that form out of an angular momentum distribution given by the 'Universal' profile of equation (14) with $\mu=1.25$ and $\lambda_{\text {gas }}$ as listed in Table 1 . We plot $\Sigma_{\text {disk }}(R)$ both as function of $R / R_{\text {vir }}$ (panels on the left) and $\log \left(R / R_{\mathrm{vir}}\right)$ (panels on the right), in order to emphasize the differences at large and small radii, respectively. 
Table 1: Global Parameters of Halos in Figures 5-8.

\begin{tabular}{rrrcccccr}
\hline \hline ID & $\begin{array}{rrrcc}N_{\text {DM }} \\
(1)\end{array}$ & $\begin{array}{r}N_{\text {gas }} \\
(2)\end{array}$ & $\begin{array}{c}\lambda_{\text {DM }} \\
(4)\end{array}$ & $\begin{array}{c}\lambda_{\text {gas }} \\
(5)\end{array}$ & $\begin{array}{c}f_{\text {DM }}^{v} \\
(6)\end{array}$ & $\begin{array}{c}f_{\text {gas }}^{v} \\
(7)\end{array}$ & $\begin{array}{c}f_{\text {gas }} \\
(8)\end{array}$ & $\begin{array}{r}\theta \\
(9)\end{array}$ \\
\hline 004 & 28584 & 27099 & 0.031 & 0.032 & 0.458 & 0.445 & 0.386 & 3.5 \\
009 & 3137 & 3196 & 0.055 & 0.057 & 0.378 & 0.375 & 0.186 & 45.7 \\
011 & 4951 & 4551 & 0.013 & 0.012 & 0.438 & 0.475 & 0.471 & 57.2 \\
012 & 3989 & 3986 & 0.041 & 0.057 & 0.440 & 0.389 & 0.239 & 0.9 \\
014 & 7394 & 6804 & 0.012 & 0.025 & 0.477 & 0.442 & 0.312 & 45.8 \\
015 & 3194 & 2901 & 0.018 & 0.016 & 0.449 & 0.458 & 0.313 & 4.4 \\
019 & 8555 & 8111 & 0.048 & 0.045 & 0.363 & 0.367 & 0.171 & 6.0 \\
021 & 3878 & 3726 & 0.050 & 0.056 & 0.390 & 0.380 & 0.186 & 15.9 \\
022 & 2882 & 2675 & 0.070 & 0.054 & 0.363 & 0.400 & 0.204 & 20.3 \\
033 & 2600 & 2656 & 0.037 & 0.048 & 0.392 & 0.402 & 0.140 & 25.3 \\
036 & 2786 & 2582 & 0.060 & 0.073 & 0.345 & 0.341 & 0.147 & 2.8 \\
052 & 2684 & 2527 & 0.035 & 0.043 & 0.389 & 0.389 & 0.245 & 29.5 \\
058 & 3652 & 3624 & 0.100 & 0.094 & 0.272 & 0.299 & 0.118 & 5.3 \\
081 & 2726 & 2653 & 0.111 & 0.119 & 0.317 & 0.333 & 0.209 & 9.6 \\
156 & 4011 & 3825 & 0.087 & 0.117 & 0.373 & 0.312 & 0.112 & 9.5 \\
170 & 3660 & 3534 & 0.027 & 0.036 & 0.415 & 0.429 & 0.269 & 8.5 \\
193 & 21030 & 20208 & 0.019 & 0.021 & 0.444 & 0.444 & 0.283 & 46.8 \\
297 & 9582 & 9218 & 0.030 & 0.049 & 0.437 & 0.401 & 0.222 & 5.1 \\
385 & 3187 & 2962 & 0.013 & 0.025 & 0.457 & 0.422 & 0.246 & 16.8 \\
582 & 17321 & 16719 & 0.048 & 0.044 & 0.414 & 0.411 & 0.310 & 30.4 \\
S0 & 63171 & 56487 & 0.016 & 0.022 & 0.461 & 0.474 & 0.451 & 61.5 \\
\hline & & & & & & & &
\end{tabular}

Note. - Global parameters of the halos whose angular momentum distributions are presented in Figures 58. Column (1) lists the halo ID. Columns (2) and (3) list the number of dark matter and gas particles, Columns (4) and (5) the spin parameters, and Columns (6) and (7) the mass fractions with negative $j_{z}^{v}$. For comparison, column (8) lists the gas mass fraction with negative $j_{z}$. Finally, column (9) lists the misalignment angle $\theta$. 